\title{
Facets of the linear ordering polytope: a unification for the fence family through weighted graphs *
}

\author{
Jean-Paul Doignon ${ }^{\mathrm{a}, *}$ Samuel Fiorini ${ }^{\mathrm{a}, \mathrm{c}, 1}$ Gwenaël Joret ${ }^{\mathrm{b}, 2}$
}

${ }^{a}$ Université Libre de Bruxelles, Département de Mathématique, c.p. 216, Boulevard du Triomphe, B-1050 Bruxelles, Belgium

${ }^{\mathrm{b}}$ Université Libre de Bruxelles, Département d'Informatique, c.p. 212, Boulevard du Triomphe, B-1050 Bruxelles, Belgium

${ }^{\mathrm{c}}$ GERAD - HEC Montréal, Bô̂te 5521, 3000 chemin de la Côte-Sainte-Catherine, Montréal (Québec) H3T 2A7, Canada

\begin{abstract}
The binary choice polytope appeared in the investigation of the binary choice problem formulated by Guilbaud (1953) and Block and Marschak (1960). It is nowadays known to be the same as the linear ordering polytope from operational research (Grötschel, Jünger and Reinelt, 1985). The central problem is to find facet-defining linear inequalities for the polytope. Fence inequalities constitute a prominent class of such inequalities (Cohen and Falmagne 1978, 1990; Grötschel, Jünger and Reinelt, 1985). Two different generalizations exist for this class: the reinforced fence inequalities (Leung and Lee, 1994; Suck, 1992) and the stability-critical fence inequalities (Koppen, 1995). Together with the fence inequalities, these inequalities form the fence family. Building on previous work on the biorder polytope (Christophe, Doignon and Fiorini, 2004), we provide a new class of inequalities which unifies all inequalities from the fence family. The proof is based on a projection of polytopes. The new class of facet-defining inequalities is related to a specific class of weighted graphs, whose definition relies on a curious extension of the stability number. We investigate this class of weighted graphs which generalize the stability-critical graphs.
\end{abstract}

Key words: binary choice polytope, linear ordering polytope, facet-defining inequalities, fence inequality, stability-critical graphs 


\section{Introduction}

A wellknown problem of mathematical psychology and economics asks for a characterization of the binary choice probabilities that are generated by random linear orderings of the alternatives (Guilbaud, 1953; Block and Marschak, 1960). This problem was turned into the search for all facet-defining inequalities of a certain (convex) polytope (Megiddo, 1977), thus dubbed the 'binary choice polytope'. On the other hand, a polytope called the 'linear ordering polytope' appeared in operations research as a tool for building an optimal solution to the linear ordering problem (Grötschel, Jünger, and Reinelt, $1985 \mathrm{a}, \mathrm{b})$. It took some time before it was realized that the two polytopes are one and the same (see for instance Suck, 1992). In both disciplines, the central problem is that of listing as much facet-defining inequalities as possiblegeometrically, one simply asks for facets. Because the problem of finding an optimal linear ordering is known to be NP-complete (Karp, 1972), there is little hope that a complete list of all facets will ever be established. Nevertheless, it is interesting to produce new facets because each of them at the same time gives a new necessary condition for binary probabilities to admit a random representation, and can also be put to good use in the optimization problem. In contrast, the multiple choice problem ashtonishingly admits an explicit solution established by Falmagne $(1978,1979)$ (for a maybe more eliciting proof, see Fiorini, 2004).

The first general scheme of facets of the binary choice vs. linear ordering polytope was discovered both in mathematical psychology and in operations research. Cohen and Falmagne $(1978,1990)$ and Grötschel et al. (1985b) indeed introduced each on their own a family of facets which surpasses the most obvious facets (although at some time in the past, the latter were thought to be the only ones). These facets are called the 'fence inequalities'. Much later, two distinct generalizations were proposed. First, introducing weights in the basic fence inequalities produced the 'reinforced fence inequalities' (Leung and Lee, 1994, followed by Suck, 1992, again illustrating parallel developments). The second generalization led through several successive steps (McLennan, 1990; Fishburn, 1990; Koppen, 1995) to 'stability-critical fence inequalities'. Here appears a marvelous connection between two distinct topics (Koppen, 1995): the latter inequalities are essentially in a one-to-one correspondence

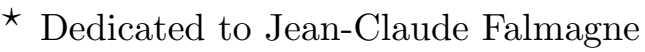

* Corresponding author

Email addresses: doignon@ulb.ac.be (Jean-Paul Doignon), sfiorini@ulb.ac.be (Samuel Fiorini), gjoret@ulb.ac.be (Gwenaël Joret).

1 During part of the project, Samuel Fiorini was a Postdoctoral Researcher of the Fonds National de la Recherche Scientifique (FNRS).

2 Gwenaël Joret is a Research Fellow of the Fonds National de la Recherche Scientifique (FNRS).
} 
with 'stability-critical graphs' (the simplest case, the fence inequality, corresponds to complete graphs).

Our contribution consists in a unification of the above two generalizations of fence inequalities via weighted graphs. To any weighted graph, we associate an inequality that is valid for the linear ordering polytope. These inequalities, which we call 'graphical inequalities', were first studied in the context of 'biorder polytopes' by Christophe, Doignon, and Fiorini (2004). When a graphical inequality defines a facet of the linear ordering polytope, the corresponding weighted graph is called a 'facet-defining graph', or FDG in short. Since FDGs generalize stability-critical graphs, we survey in Section 6 known results about the latter graphs. In particular, we emphasize the role of the 'defect' in attempts to classify stability-critical graphs. Section 7 is devoted to basic results on FDGs, some of them taken from Christophe, Doignon, and Fiorini (2004). The following section introduces the 'defect' of a FDG and establishes several of its properties. It concludes with the first steps into the classification of FDGs with small defect.

To summarize, our contribution goes beyond providing a common generalization for the fence family. We also establish a list of properties of the corresponding FDGs. In this respect, our work is parallel to that of Lipták and Lovász $(2000,2001)$ who also investigate a generalization of stability-critical graphs in connection with (other) polytopes. Before focusing on FDGs, we formally describe in Sections 2 and 3 the fence family and the graphical inequalities, respectively. Then in Section 4 we collect prerequisites on biorders, relying on Doignon, Ducamp, and Falmagne (1984). Section 5 introduces a projection from the linear ordering polytope onto the biorder polytope. The projection is then used to prove that a graphical inequality is facet-defining for the linear ordering polytope if and only if it is facet-defining for the biorder polytope, except in one particular case.

This paper is heavily influenced by the work of Jean-Claude Falmagne. The senior author (J.-P. D.) was exposed by him to biorders in 1982 and to the binary choice polytope in 1988. All three authors are glad to dedicate their present contribution to Jean-Claude.

\section{Background and the Fence Family}

Let $X, Y$ be finite sets, and let $R$ denote a relation from $X$ to $Y$. As relations are always considered as sets of ordered pairs in this paper, $R$ is a subset of $X \times Y$. We often use $i j$ as an abbreviation for $(i, j)$ and write $i R j$ when the pair $i j$ belongs to the relation $R$. In order to encode $R$ geometrically, we resort to the real vector space $\mathbb{R}^{X \times Y}$, which has one coordinate per element 
of $X \times Y$. The coordinate of the pair $i j$ is denoted by $x_{i j}$. The characteristic vector of $R$ is the vector $x^{R}$ in $\mathbb{R}^{X \times Y}$ such that $x_{i j}^{R}=1$ if $i j \in R$ and $x_{i j}^{R}=0$ otherwise.

Now let $Z$ be a third finite set. A linear ordering on $Z$ is a reflexive, transitive, antisymmetric and complete relation on $Z$, i.e., from $Z$ to $Z$. The binary choice polytope or linear ordering polytope is defined as the convex hull in $\mathbb{R}^{Z \times Z}=\mathbb{R}^{Z^{2}}$ of the characteristic vectors $x^{L}$ of all linear orderings $L$ on $Z$. We denote it by $P_{\mathrm{LO}}^{Z}$. Formally, we have

$$
P_{\mathrm{LO}}^{Z}=\operatorname{conv}\left\{x^{L} \in \mathbb{R}^{Z^{2}} \mid L \text { is a linear ordering on } Z\right\} .
$$

The linear ordering polytope has precisely one vertex per linear ordering on $Z$. Note that the whole polytope lies inside the affine subspace defined by the equations $x_{i i}=1$ for $i \in Z$ and $x_{i j}+x_{j i}=1$ for $i, j \in Z, i \neq j$. Because these equations form a complete and irredundant system of equations for the polytope, we have $\operatorname{dim} P_{\mathrm{LO}}^{Z}=|Z|(|Z|-1) / 2$. We remark that besides the obvious symmetries derived from permutations of the base set $Z$ and arc reversal, the linear ordering polytope admits 'strange' symmetries found by McLennan (1990) and Bolotashvili, Kovalev, and Girlich (1999). The full group of symmetries was characterized by Fiorini (2001).

Classes of facet-defining inequalities for the linear ordering polytope are now described. Because they are all related to the fence inequalities (defined below), we collectively refer to them as the fence family. In the rest of the section, $X$ and $Y$ are disjoint subsets of $Z$ with the same cardinality, and $f$ is a bijective mapping from $X$ to $Y$. A fence inequality is any inequality of the form

$$
\sum_{i \in X} x_{i f(i)}-\sum_{\substack{i \in X, j \in Y \\ j \neq f(i)}} x_{i j} \leq 1
$$

Notice that, traditionnally, the fence inequality is written in another, equivalent form. This inequality was independently discovered by Grötschel et al. (1985a) and by Cohen and Falmagne (1990). Although the latter reference was published five years after the former, the working paper version dates back to 1978.

Proposition 1 (Grötschel et al., 1985a) The fence inequality (2) defines a facet of the linear ordering polytope $P_{\mathrm{LO}}^{Z}$ whenever $|Z| \geq 2|X|=2|Y| \geq 6$.

A first idea to generalize the fence inequalities is to multiply all the terms of the form $x_{i f(i)}$ in Inequality (2) by an integer $t$ with $1 \leq t \leq|X|-2$. The resulting inequality,

$$
\sum_{i \in X} t x_{i f(i)}-\sum_{\substack{i \in X, j \in Y \\ j \neq f(i)}} x_{i j} \leq \frac{t(t+1)}{2},
$$


is called a reinforced fence inequality. Although these inequalities were given a name by Leung and Lee (1994), they were implicitly known before as special cases of Gilboa's 'diagonal inequalities' (Gilboa, 1990, working paper of 1985). They were independently discovered also by Suck (1992).

Proposition 2 (Leung and Lee, 1994; Suck, 1992) The reinforced fence inequality (3) defines a facet of the linear ordering polytope $P_{\mathrm{LO}}^{Z}$ whenever $|Z| \geq 2|X|=2|Y| \geq 6$ and $1 \leq t \leq|X|-2$.

A second generalization of the fence inequalities, due to Koppen (1995), arises when the complete graph implicit in the structure of the fence inequality is replaced by an arbitrary graph. Let thus $G$ be any graph whose vertex set $V(G)$ equals $X$ (for graph terminology, we usually follow Diestel, 2000). With $\alpha(G)$ denoting the stability number of $G$, the inequality

$$
\sum_{v \in V(G)} x_{v f(v)}-\sum_{\{v, w\} \in E(G)}\left(x_{v f(w)}+x_{w f(v)}\right) \leq \alpha(G)
$$

is easily seen to be valid for the linear ordering polytope. Koppen (1995) gave the following characterization of the graphs $G$ for which Inequality (4) is facet-defining.

Proposition 3 (Koppen, 1995) Inequality (4) defines a facet of the linear ordering polytope if and only if $G$ is the one-vertex graph or $G$ has at least three vertices, is connected and stability-critical.

A graph $G$ without isolated vertex is said to be stability-critical if its stability number increases whenever an edge is removed from its edge set. When $G$ satisfies the conditions of Proposition 3, we call Inequality (4) a stabilitycritical fence inequality.

Observe that when $G$ is a complete graph with at least three vertices, Inequality (4) is a fence inequality. Hence stability-critical fence inequalities generalize fence inequalities. Two more special cases of stability-critical fence inequalities have been considered in the literature. The first special case occurs when $G$ is an odd cycle. The corresponding inequalities were discovered independently by Grötschel et al. (1985a), McLennan (1990) and Fishburn (1990). The second special case, which subsumes the first, occurs when $G$ is the graph $C_{n}^{\ell}$ with vertex set $V=\{1,2, \ldots, n\}$ and edge set

$$
E=\{\{i, j\} \mid i, j \in V, 0<\min \{|i-j|,|k-i-j|\} \leq \ell\},
$$

and with $3 \leq 2 \ell+1 \leq n$. The corresponding inequalities were investigated independently by Bolotashvili (1987) under the name $(n, \ell+1)$-fence inequalities, and by Koppen (1991). It is known that $\alpha\left(C_{n}^{\ell}\right)=\lfloor n /(\ell+1)\rfloor$ and that $C_{n}^{\ell}$ is stability-critical if and only if $\ell+1$ divides $n+1$. Apparently, Bolotashvili 
(1987) showed that the $(n, \ell+1)$-fence inequalities define facets of the linear ordering polytopes, provided that $\ell+1$ divides $n+1$.

We remark that by applying (nonobvious) symmetries of the linear ordering polytope to the facet-defining inequalities given above, one obtains new facet-defining inequalities. Some of them were studied in the litterature, as for instance the augmented fence inequalities of McLennan (1990) and Leung and Lee (1994), and the augmented reinforced fence inequalities of Leung and Lee (1994).

\section{Graphical Inequalities}

In the preceding section, we have seen two different generalizations of the fence inequalities. The first changes the coefficients of the positive terms and the second changes the structure of the inequality by substituting any graph for the complete graph. It is quite natural to combine both generalizations, which is precisely what is done in this section.

A weighted graph is a pair $(G, \mu)$ where $G$ is a graph and $\mu$ is a function assigning an integral weight $\mu(v)$ to each vertex $v$ of $G$. Let $S$ be any subset of the vertex set of $G$. We denote by $\mu(S)=\sum_{v \in S} \mu(v)$ the total weight of $S$. The worth (or net weight) of $S$ is the difference between the total weight of $S$ and the number of edges in the subgraph of $G$ induced by $S$. This number of edges, denoted as $\|G[S]\|$ in Diestel (2000), will be given here by the simpler notation $\|S\|$. Thus the worth of $S$ equals

$$
w(S)=\mu(S)-\|S\|
$$

If $S$ is of maximum worth amongst subsets of $V(G)$ we say that $S$ is tight. We define $\alpha(G, \mu)$ to be the worth of a tight set in $(G, \mu)$. That is, we let

$$
\alpha(G, \mu)=\max _{S \subseteq V(G)} w(S) .
$$

When $\mu=\mathbb{1}$, i.e., when the weight of each vertex is 1 , we have $\alpha(G, \mathbb{1})=\alpha(G)$. Hence the parameter $\alpha(G, \mu)$ can be considered as a generalization of the stability number of a graph to weighted graphs.

Let $(G, \mu)$ be a weighted graph whose vertex set $V(G)$ equals $X$. We again assume that $X$ and $Y$ are disjoint subsets of $Z$, with $f$ a bijection from $X$ to $Y$. The graphical inequality of $(G, \mu)$ reads

$$
\sum_{v \in V(G)} \mu(v) x_{v f(v)}-\sum_{\{v, w\} \in E(G)}\left(x_{v f(w)}+x_{w f(v)}\right) \leq \alpha(G, \mu) .
$$


Because of the choice of the right-hand side, the inequality is always valid for the linear ordering polytope $P_{\mathrm{LO}}^{Z}$. When $\mu=1$, it is identical to Inequality (4). Morever, when $G$ is a complete graph and $\mu=t 1$ with $1 \leq t \leq|X|-2$, the graphical inequality is a reinforced fence inequality.

We say that a weighted graph $(G, \mu)$ is facet-defining when its graphical inequality defines a facet of the linear ordering polytope. A vertex of a weighted graph is said to be degenerated if both its weight and its degree equal zero. In order to avoid trivial cases, we always assume that a weighted graph has no degenerated vertex. We think that understanding the structure of facetdefining graphs, in short FDGs, is a nice and important research problem. By Proposition 3, this class contains all connected stability-critical graphs except the complete graph $K_{2}$. We survey some important results on stability-critical graphs in Section 6, and adapt these results to the more general case of facetdefining graphs in Sections 7 and 8. We also provide results about FDGs which are of a new type.

Before starting to investigate FDGs, we need to establish when a graphical inequality defines a facet of the linear ordering polytope. To this aim, we make use of another polytope, namely the 'biorder polytope'. In the next two sections, we remind the reader about biorders and the definition and some properties of the biorder polytope.

\section{Biorders and the Biorder Polytope}

Let $X$ and $Y$ be two finite sets. A relation from $X$ to $Y$ is a biorder when

$$
i B j \text { and } k B \ell \text { imply } i B \ell \text { or } k B j
$$

for all elements $i, k \in X$ and $j, \ell \in Y$. While biorders received various names, for instance 'Guttman scales' (after Guttman, 1944), 'Ferrers relations' (Riguet, 1951), 'bi-quasi-series' (Ducamp and Falmagne, 1969), the term comes from Doignon et al. (1984) (where the case of infinite sets $X$ and $Y$ is also considered). The number of biorders from $X$ to $Y$ is a function of only $|X|$ and $|Y|$, which is investigated in Christophe, Doignon, and Fiorini (2003).

The biorder polytope $P_{\mathrm{Bio}}^{X \times Y}$ was introduced in Christophe et al. (2004), with a definition similar to that of the linear ordering polytope. Each biorder $B$ from $X$ to $Y$ is encoded by its characteristic vector $x^{B}$, considered as an element of the space $\mathbb{R}^{X \times Y}$ (points in this space have one coordinate $x_{i j}$ for each ordered pair $i j$ in $X \times Y)$. The convex hull of all points $x^{B}$ in $\mathbb{R}^{X \times Y}$, for $B$ any biorder from $X$ to $Y$, is the biorder polytope $P_{\mathrm{Bio}}^{X \times Y}$. The biorder polytope $P_{\mathrm{Bio}}^{X \times Y}$ has dimension $|X| \cdot|Y|$. 
The graphical inequality has an even more natural definition for the biorder polytope $P_{\mathrm{Bio}}^{X \times Y}$ than for the linear ordering polytope (cf. Equation (8)). Assume the weighted graph $(G, \mu)$ is such that $V(G)=X$, and consider any bijective mapping $f: X \rightarrow Y$. The graphical inequality of $(G, \mu)$ for $P_{\text {Bio }}^{X \times Y}$ reads

$$
\sum_{v \in V(G)} \mu(v) x_{v f(v)}-\sum_{\{v, w\} \in E(G)}\left(x_{v f(w)}+x_{w f(v)}\right) \leq \alpha(G, \mu) .
$$

The following results are adapted from Christophe et al. (2004). The graphical inequality is valid for $P_{\mathrm{Bio}}^{X \times Y}$. It defines a facet if and only if the tight sets of $(G, \mu)$ satisfy a technical condition that we will formulate in Proposition 4 after having introduced some concepts. Let $(G, \mu)$ be a weighted graph. We denote by $E(S)$ the collection of edges contained in the set $S$ of vertices. To each tight set $T$ of $(G, \mu)$, we associate the affine equation

$$
\sum_{v \in T} y_{v}+\sum_{e \in E(T)} y_{e}=\alpha(G, \mu)
$$

We thus form the system of $(G, \mu)$, also described as $\mathcal{T} \cdot \mathcal{Y}=\mathcal{A}$, where the rows of the matrix $\mathcal{T}$ correspond to tight sets of $(G, \mu)$, the vector $\mathcal{Y}$ contains the real unknowns $y_{v}$ and $y_{e}$ for $v \in V(G)$ and $e \in E(G)$, and $\mathcal{A}=[\alpha(G, \mu) \alpha(G, \mu) \ldots \alpha(G, \mu)]^{t}$.

Proposition 4 (Christophe et al., 2004) A weighted graph $(G, \mu)$ is facetdefining (that is, $(G, \mu)$ is a FDG) if and only if it has at least three vertices and the system of $(G, \mu)$ has a unique solution.

The vector $y$ defined by $y_{v}=\mu(v)$ and $y_{e}=-1$ for all $v \in V(G), e \in E(G)$ is always a solution to the system of $(G, \mu)$, so we require in Proposition 4 that there is no other solution.

Assuming some relationships among $X, Y$ and $Z$, we now proceed to show that the graphical inequality is facet-defining for $P_{\text {Bio }}^{X \times Y}$ if and only if it is facetdefining for $P_{\mathrm{LO}}^{Z}$. A projection from $P_{\mathrm{LO}}^{Z}$ onto $P_{\mathrm{Bio}}^{X \times Y}$ will be instrumental.

\section{Projection of the Linear Ordering Polytope onto the Biorder Polytope}

Let $Z$ be any finite set, and $X, Y$ be disjoint, nonempty subsets of $Z$. To any relation $R$ on $Z$ we associate the induced relation from $X$ to $Y$, which is the intersection $R \cap(X \times Y)$. As we will now show, linear orderings on $Z$ are then exactly mapped onto the biorders from $X$ to $Y$. 
Proposition 5 Let $X, Y$ and $Z$ be as above. Any linear ordering on $Z$ induces a biorder from $X$ to $Y$. Conversely, every biorder from $X$ to $Y$ is induced by a linear ordering on $Z$.

PROOF. The intersection of any linear ordering on $Z$ with $X \times Y$ is a biorder from $X$ to $Y$, as easily seen. Hence the first part of the proposition holds. To show the second part, let $B$ be a biorder from $X$ to $Y$. Then the relation $R$ on $Z$ obtained from $B$ by adding all pairs $j i \in Y \times X$ with $i j \notin B$ is acyclic. Hence $R$ is contained in some linear ordering $L$ on $Z$. By the choice of $R$, the biorder from $X$ to $Y$ induced by $L$ is exactly $B$.

We now build a projection from the linear ordering polytope $P_{\mathrm{LO}}^{Z}$ onto the biorder polytope $P_{\mathrm{Bio}}^{X \times Y}$. First define the linear projection

$$
\pi: \mathbb{R}^{Z^{2}} \rightarrow \mathbb{R}^{X \times Y}: x \mapsto x^{\prime}=\pi(x),
$$

where $x_{i j}^{\prime}=x_{i j}$ for $i j \in X \times Y$. From Proposition 5, we see at once that $\pi$ maps the vertex set of the linear ordering polytope onto the vertex set of the biorder polytope. Indeed, $\pi$ maps a vertex $x^{L}$ of the linear ordering polytope onto the vertex $x^{B}$ of the biorder polytope, where $B=L \cap(X \times Y)$ is the biorder induced by $L$. As a consequence, $\pi$ maps the linear ordering polytope $P_{\mathrm{LO}}^{Z}$ onto the biorder polytope $P_{\mathrm{Bio}}^{X \times Y}$. By the proof of Proposition 5, the vertices of the linear ordering polytope which are mapped by $\pi$ to a given vertex $x^{B}$ of the biorder polytope correspond to the linear extensions of an acyclic orientation of the complete bipartite graph with color classes $X$ and $Y$ determined by $B$.

We now switch to a more general setting in order to state and prove a lemma which is instrumental for showing the main result of this section. Let $P$ and $Q$ be two polytopes and let $\dot{\rho}: P \rightarrow Q$ denote a projection of polytopes, that is, the restriction to $P$ of an affine map $\rho$ from the space in which $P$ is defined to the space in which $Q$ is defined, mapping $P$ onto $Q$. The projection $\dot{\rho}$ yields a lifting of the faces of $Q$ to the faces of $P$ : for every face $F$ of $Q$ the preimage $\dot{\rho}^{-1}(F)=\{x \in P \mid \rho(x) \in F\}$ is a face of $P$. Consider a face $F$ of $Q$. The plank of $F$ is the vector subspace defined by

$$
\operatorname{plank} F=\operatorname{lin}\{q-p \mid p, q \in P \text { and } \rho(p)=\rho(q) \in F\} .
$$

Note that the the plank itself depends on a choice of origin in the ambient space of $P$, but its dimension is always the same. As we now show, this vector subspace is useful in computing the dimension of the preimage of a face.

Lemma 6 For any face $F$ of $Q$, we have

$$
\operatorname{dim} \dot{\rho}^{-1}(F)=\operatorname{dim} F+\operatorname{dim} \text { plank } F .
$$


PROOF. Let $W$ and $V$ respectively denote the two affine subspaces spanned by $F$ and its preimage, respectively. Let $o$ be a point in the relative interior of $\dot{\rho}^{-1}(F)$. Taking $o$ as an origin in $V$ and its image $\rho(o)$ as an origin in $W$, we can regard $V$ and $W$ as vector spaces. The affine map $\rho$ restricts to a linear mapping $R$ from $V$ onto $W$. As is easily verified, the plank of $F$ computed with $O$ as origin is simply the kernel of $R$. The lemma then follows from the well-known equation $\operatorname{dim} V=\operatorname{dim} W+\operatorname{dim} \operatorname{ker} R$.

Before turning to the main result of this section, we note the following lemma.

Lemma 7 If the preimage $\dot{\rho}^{-1}(F)$ of a face $F$ of $Q$ is a facet of $P$, then $F$ is itself a facet of $Q$.

PROOF. If $F$ is not a facet of $Q$ then there exists a facet $F^{\prime}$ of $Q$ which properly contains $F$. Now the preimage of $F^{\prime}$ properly contains the preimage of $F$, so the preimage of $F^{\prime}$ equals $Q$, contradicting the fact that $\dot{\rho}$ is surjective.

We now go back to our initial case, where $P=P_{\mathrm{LO}}^{Z}, Q=P_{\mathrm{Bio}}^{X \times Y}$ and $\rho=\pi$. Let $\dot{\pi}$ denote the restriction of $\pi$ to $P_{\mathrm{LO}}^{Z}$. Thus $\dot{\pi}$ plays the role of $\dot{\rho}$. Moreover, as in Sections 2 and 3, we suppose $|X|=|Y|$ and that some bijection $f: X \rightarrow Y$ is given.

Proposition 8 Let $(G, \mu)$ be a weighted graph such that Inequality (8) defines a facet $F$ of $P_{\mathrm{Bio}}^{X \times Y}$. Then the preimage of $F$ under $\dot{\pi}$ is a facet of $P_{\mathrm{LO}}^{Z}$, unless $(G, \mu)=\left(K_{2}, \mathbb{1}\right)$.

PROOF. Since the assertion is easily verified when $G$ has at most two vertices, we can assume that $G$ has at least three vertices. Set $q=|Z|$, $m=|X|=|Y|$. In virtue of the trivial lifting lemmas for linear ordering polytopes (Grötschel et al., 1985a), we may assume $q=2 \mathrm{~m}$, that is, $X$ and $Y$ partition $Z$. It suffices then to prove

$$
\operatorname{dim} \text { plank } F \geq \operatorname{dim} P_{\mathrm{LO}}^{Z}-\operatorname{dim} P_{\mathrm{Bio}}^{X \times Y}=\frac{q(q-1)}{2}-m^{2} .
$$

Indeed, this inequality together with Lemma 6 implies that the dimension of the preimage of $F$ is at least that of a facet of the linear ordering polytope. On the other hand, the preimage of $F$ is not the whole linear ordering polytope because $F$ is a proper face and $\pi$ is surjective, hence $\dot{\pi}^{-1}(F)$ is a facet of $P_{\mathrm{LO}}^{Z}$.

Notice that the right-hand side of Equation (14) is the number of unordered pairs $\left\{k, k^{\prime}\right\}$ such that $k, k^{\prime} \in X$ or $k, k^{\prime} \in Y$. We denote by $e_{k k^{\prime}}$ the vector in the canonical basis of $\mathbb{R}^{Z^{2}}$ that corresponds to the pair $k k^{\prime}$. Let us show 
that for all $i, i^{\prime} \in X$ with $i \neq i^{\prime}$, we get $e_{i i^{\prime}}-e_{i^{\prime} i} \in \operatorname{plank} F$. As a similar argument can be given for all $j, j^{\prime} \in Y$ with $j \neq j^{\prime}$, we are done because altogether these $2 \cdot m(m-1)$ vectors generate a linear subspace of dimension $2 \cdot(m(m-1) / 2)=q(q-1) / 2-m^{2}$.

Case 1: $\left\{i, i^{\prime}\right\} \in E(G)$. By Proposition 11(C3) in Christophe et al. (2004), or by Proposition 11(C4) of Section 7, there exists a tight set $S$ avoiding both $i$ and $i^{\prime}$. Pick any linear ordering $M$ on $S$ and list the elements of $S$ by increasing ranks as $s_{1}, s_{2}, \ldots, s_{\ell}$. Then $B=\{x f(y) \mid x y \in M\}$ is a biorder from $X$ to $Y$ whose characteristic vector is a vertex of $F$, according to Proposition 6 in Christophe et al. (2004). Any linear ordering $L$ on $Z$ which has $Y \backslash f(S)$ as initial set, $X \backslash S$ as final set and which satisfies $s_{1} L f\left(s_{1}\right) L s_{2} L f\left(s_{2}\right) L \ldots L s_{\ell} L f\left(s_{\ell}\right)$ induces $B$ on $X \times Y$. There exist two such linear orderings $L_{1}$ and $L_{2}$ with $L_{1} \backslash L_{2}=\left\{i i^{\prime}\right\}$ and $L_{2} \backslash L_{1}=\left\{i^{\prime} i\right\}$. It follows $x^{L_{1}}-x^{L_{2}}=e_{i i^{\prime}}-e_{i^{\prime} i} \in \operatorname{plank} F$.

Case 2: $\left\{i, i^{\prime}\right\} \notin E(G)$. There exists some tight set $S$ containing exactly one vertex in $\left\{i, i^{\prime}\right\}$. This is true because if no such $S$ existed, the system in Equation (13) of Christophe et al. (2004) would not have a unique solution, contradicting the fact that $F$ is a facet (or see Proposition 11(C7) in Section 7). Without loss of generality, we assume $i \in S$ and $i^{\prime} \notin S$. Let $M$ be any linear ordering on $S$ with $i$ as its maximum, then $B=\{x f(y) \mid x y \in M\} \cup\left\{i^{\prime} f(i)\right\}$ is a biorder from $X$ to $Y$ such that $x^{B}$ is a vertex of $F$. The argument then goes as in the first case above.

Using Lemma 7, we can easily show that the converse of Proposition 8 also holds. Summarizing, we see that the following corollary holds.

Corollary 9 A graphical inequality is facet-defining for the linear ordering polytope $P_{\mathrm{LO}}^{Z}$ if and only if it is facet-defining for the biorder polytope $P_{\mathrm{Bio}}^{X \times Y}$, except if the underlying weighted graph is $\left(K_{2}, 1\right)$.

\section{$6 \quad$ Stability-critical graphs}

In this section, we briefly survey important results concerning stability-critical graphs, thus complementing the report of Koppen (1995) (Section 7). We refer the reader to Lovász and Plummer (1986) (pages 445-456) and Lovász (1993) (pages 64-65) for a more detailed account.

We remind the reader that a graph is stability-critical if it has no isolated vertices and removing any of its edges increases its stability number. The class of stability-critical graphs was first studied by Erdős and Gallai (1961). 
They introduced the defect $\delta(G)=|V(G)|-2 \alpha(G)$ of a graph $G$ and proved its nonnegativity when $G$ is stability-critical. The defect $\delta$ is a key parameter in the theory of stability-critical graphs. Hajnal (1965) established an upper bound of $\delta+1$ on the degree of a vertex and Surányi (1975b) proved that equality in the previous bound can occur for at most $\delta+2$ vertices if $\delta>1$. Sewell and Trotter (1993) also proved that every stability-critical graph with defect at least two contains an odd subdivision of $K_{4}$, that is, the graph $K_{4}$ where each edge is replaced by a path with an odd number of edges.

From a general viewpoint, stability-critical graphs exhibit many different structures and a satisfying characterization seems out of reach. Nevertheless, more insight was obtained by considering them for a fixed defect. Indeed, let $G$ be a connected stability-critical graph (note that the assumption of connectedness is not really restrictive, since a non connected stability-critical graph consists of connected stability-critical components). If $\delta(G)=0$, the theorem of Hajnal (recalled in previous paragraph) implies $G=K_{2}$. For $\delta(G)=1$, it also implies that $G$ is either a path or a cycle. Because paths and even cycles have defect $<1$ (and also are not stability-critical, except for $K_{2}$ ), $G$ must be an odd cycle or, equivalently, an odd subdivision of $K_{3}$. Andrásfai (1967) proved that $\delta(G)=2$ occurs exactly when $G$ is an odd subdivision of $K_{4}$. More generally, for each fixed natural number $\delta$ there is a finite set of graphs such that if $\delta(G)=\delta$ then $G$ is an odd subdivision of one of those. This was first proved for $\delta=3$ by Surányi (1975b) and later for all $\delta \geq 1$ by Lovász (1978).

As seen in the previous paragraph, the odd subdivision of a graph is used as a closure operation when characterizing stability-critical graphs of a given defect. In its simplest form, an odd subdvision only trisects one edge, that is, it replaces the edge by a path composed of three edges. Thus, an odd subdivision can be seen as a composition of a certain number of trisections. It turns out that trisecting an edge is only but a special case of a more general method to construct a connected stability-critical graph by 'gluing' two smaller ones. In order to describe it, we need the fact that connected stability-critical graphs are also 2-connected (Lovász, 1993). Let $G_{1}$ and $G_{2}$ be two connected stabilitycritical graphs other than $K_{2}$ and choose an edge $\{a, b\}$ of $G_{1}$ and a vertex $c$ of $G_{2}$. Define the graph $G$ upon basis of $G_{1}$ and $G_{2}$ as follows (an example is given in Figure 1):

- take the disjoint union of $G_{1}$ and $G_{2}$,

- remove the edge $\{a, b\}$,

- make each neighbor of $c$ adjacent to exactly one vertex of $\{a, b\}$, ensuring that $a$ and $b$ have each one at least one such neighbor, and

- remove the vertex $c$.

Observe that $G$ is 2-connected but not 3-connected, since removing the vertices $a$ and $b$ disconnects $G$. One can check also that the equality $\delta(G)=\delta\left(G_{1}\right)+$ 

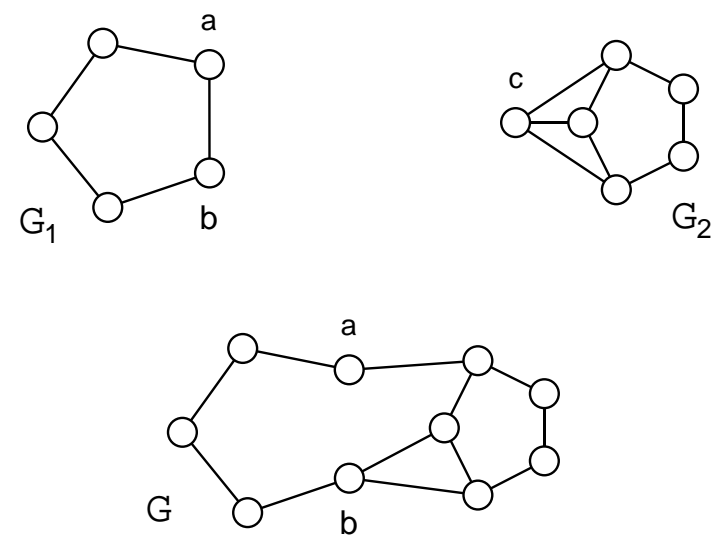

Fig. 1. An example of the construction of new stability-critical graphs.

$\delta\left(G_{2}\right)-1$ holds. When we let $G_{2}=K_{3}$, this construction is equivalent to trisecting the edge $\{a, b\}$ of $G_{1}$ and does not affect its defect (that is, $\delta(G)=$ $\delta\left(G_{1}\right)$ ). Plummer (1967) first studied this construction when $G_{1}$ is a complete graph and later Wessel (1970a) extended its work by showing that, in the above construction, the graph $G$ must also be stability-critical and moreover that any connected non 3-connected stability-critical graph different from $K_{2}$ arises in this way.

We conclude this section by mentioning other references concerning stabilitycritical graphs: Beineke et al. (1967); Erdős et al. (1964); Harary and Plummer (1967); Sewell and Trotter (1995); Surányi (1975a, 1978, 1980); Wessel (1968, 1975, 1978, 1970b); Zhu (1989).

\section{$7 \quad$ Facet-defining graphs}

Now considering weighted graphs as in Section 3, we will generalize stabilitycritical graphs. By Koppen's result (Proposition 3), all connected stabilitycritical graphs with at least three vertices (with a constant weight 1 on all vertices) are such that their graphical inequality defines a facet of the linear ordering polytope $P_{\mathrm{LO}}^{Z}$. Remember that the weighted graphs for which the graphical inequality defines a facet of $P_{\mathrm{LO}}^{Z}$ are the facet-defining graphs, or FDGs for short. Corollary 9 states that exactly the same weighted graphs produce a facet-defining inequality of the biorder polytope $P_{\mathrm{Bio}}^{X \times Y}$ (with some relationships between $Z$ and $X, Y)$, except for the trivial case $\left(K_{2}, \mathbb{1}\right)$. In this section we recall facts about FDGs obtained in Christophe et al. (2004) and describe a first bunch of new results. The next section provides more contributions about these graphs.

Let $(G, \mu)$ be a weighted graph. Proposition 4 in Section 4 indicates exactly when $(G, \mu)$ is facet-defining, in terms of the system $\mathcal{T} \cdot \mathcal{Y}=\mathcal{A}$ of $(G, \mu)$. Here 
is a reformulation of the condition.

Corollary 10 A weighted graph $(G, \mu)$ is facet-defining if and only if it has at least three vertices and for each nonzero valuation $\lambda: V(G) \cup E(G) \rightarrow \mathbb{Z}$ there is a tight set $T$ of $(G, \mu)$ with

$$
\sum_{v \in T} \lambda(t)+\sum_{e \in E(T)} \lambda(e) \neq 0
$$

PROOF. By Proposition $4,(G, \mu)$ is facet-defining if and only if $G$ has at least three vertices and moreover the system $\mathcal{T} \cdot \mathcal{Y}=\mathcal{A}$ has only one solution. The latter condition amounts to: the homogeneous system $\mathcal{T} \cdot \mathcal{Y}=0$ has only the zero solution. In turn, this is equivalent to: the only linear combination of column vectors of $\mathcal{T}$ which produces the zero vector has only null coefficients. By contraposing, we get the claim.

Corollary 10 is useful to derive necessary conditions for a weighted graph to be facet-defining, as illustrated in the next proposition.

Proposition 11 Let $(G, \mu)$ be a FDG. Then the following conditions hold:

(C1) $G$ is 2-connected;

(C2) for all $v \in V(G)$, we have $1 \leq \mu(v) \leq \operatorname{deg}(v)-1$;

(C3) for all $\{v, w\} \in E(G)$, there is a tight set containing $v$ and $w$;

(C4) for all $\{v, w\} \in E(G)$, there is a tight set containing neither $v$ nor $w$;

(C5) for all $\{v, w\} \in E(G)$, there is a tight set containing $v$ and not $w$;

(C6) for all $v, w \in V(G),\{v, w\} \notin E(G)$, there is a tight set containing either both vertices $v$ and $w$ or none of them;

(C7) for all $v, w \in V(G),\{v, w\} \notin E(G)$, there is a tight set containing exactly one vertex of $\{v, w\}$.

PROOF. (C1)-(C4) were already proved in Christophe et al. (2004) and we refer to it for $(\mathrm{C} 1)$ and $(\mathrm{C} 2)$. We prove $(\mathrm{C} 3)-(\mathrm{C} 7)$ by using Corollary 10 with an appropriate choice for the valuation $\lambda$ (this is a new proof for $(\mathrm{C} 3)-(\mathrm{C} 4)$ ).

(C3) Set $\lambda(\{v, w\})=1$ and $\lambda$ to zero elsewhere.

(C4) Set $\lambda(v)=\mu(v)-\alpha(G, \mu), \lambda(w)=\mu(w)-\alpha(G, \mu), \lambda(\{v, w\})=\alpha(G, \mu)-$ $1, \lambda(u)=\mu(u)$ for every $u \in V(G) \backslash\{v, w\}$ and $\lambda(e)=-1$ for every $e \in E(G) \backslash\{\{v, w\}\}$.

(C5) Set $\lambda(v)=1, \lambda(\{v, w\})=-1$ and $\lambda$ to zero elsewhere.

(C6) Set $\lambda(v)=\mu(v)-\alpha(G, \mu), \lambda(w)=\mu(w)-\alpha(G, \mu), \lambda(u)=\mu(u)$ for every $u \in V(G) \backslash\{v, w\}$ and $\lambda(e)=-1$ for every $e \in E(G)$.

(C7) Set $\lambda(v)=1, \lambda(w)=-1$ and $\lambda$ to zero elsewhere. 

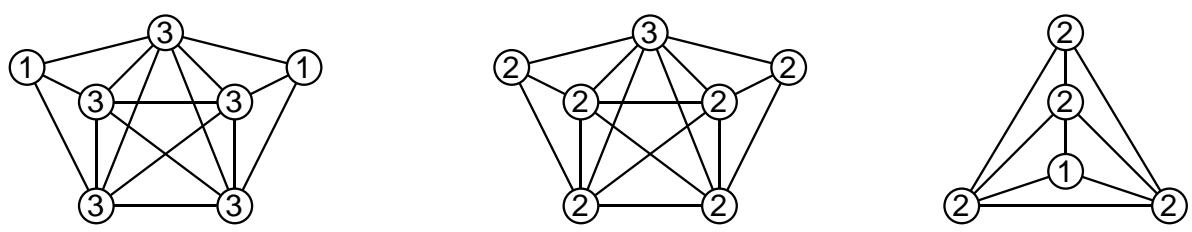

Fig. 2. Three specific FDGs.

It can easily be checked that each time the specific valuation $\lambda$ ensures by Corollary 10 the existence of a tight set with the desired property.

There exist FDGs showing that Conditions (C6) and (C7) of Proposition 11 cannot be strengthened as in (C3), (C4) and (C5), examples are given on Figure 2: in the left graph there is no tight set containing the two vertices with unit weight, in the central one there is no tight set avoiding the two vertices with degree 3 , and in the right one there is no tight set containing the unit weight vertex and not the only vertex nonadjacent to it.

Proposition 3 states that all stability-critical graphs together with the weight function $\mathbb{1}$ are facet-defining graphs, except for $K_{2}$. Many more FDGs are derived by applying together Corollary 9 and techniques of Christophe et al. (2004), as we now explain. Let $(G, \mu)$ be a connected weighted graph. We say that $(G, \mu)$ is a special facet-defining graph, abbreviated SFDG, if for each $v \in V(G)$ we have $1 \leq \mu(v) \leq \operatorname{deg}(v)-1$ and for each $v, w_{1}, \ldots, w_{k} \in V(G)$ such that $k=\mu(v)$ and $v w_{1}, \ldots, v w_{k} \in E(G)$, there exists a tight set $T$ of $(G, \mu)$ containing $v, w_{1}, \ldots, w_{k}$. These graphs are all FDGs, as shown by the following proposition.

Proposition 12 (Christophe et al., 2004) A SFDG is facet-defining, that is, any $S F D G$ is a FDG.

We note that in particular connected stability-critical graphs other than $K_{2}$ equipped with the weight function $\mathbb{1}$ are SFDG by Proposition 11(C3) and by Proposition 3. There exist FDGs which are not SFDGs, the three graphs in Figure 2 for instance.

We complete this section by reporting an interesting result from Christophe et al. (2004) linking a weighted graph $(G, \mu)$ to the one obtained by taking $\operatorname{deg}-\mu$ as weight function, where deg assigns to each vertex its degree. We let $\|G\|=|E(G)|$.

Proposition 13 (Christophe et al., 2004) Let $(G, \mu)$ be a weighted graph. Then the following holds:

- $\alpha(G, \operatorname{deg}-\mu)=\alpha(G, \mu)-(\mu(V(G))-\|G\|)$,

- a set $T \subseteq V(G)$ is tight in $(G, \mu)$ if and only if $V(G) \backslash T$ is tight in 
$(G, \operatorname{deg}-\mu)$, and

- $(G, \mu)$ is facet-defining if and only if $(G, \operatorname{deg}-\mu)$ is facet-defining.

In order to illustrate Proposition 13, we remark that the central graph in Figure 2 is obtained from the left one using the described modification of the weights.

\section{The defect of facet-defining graphs}

The defect of a graph $G$ was defined in Section 6 as $\delta(G)=|V(G)|-2 \alpha(G)$. We generalize the concept to weighted graphs by letting the defect of $(G, \mu)$ be

$$
\delta(G, \mu)=\mu(V(G))-2 \alpha(G, \mu)
$$

(when $\mu=\mathbb{1}$, we have $\delta(G, \mu)=\delta(G)$ ). We first observe an interesting fact.

Proposition $14 \operatorname{Let}(G, \mu)$ be a weighted graph. Then $\delta(G, \mu)=\delta(G, \operatorname{deg}-\mu)$.

PROOF. The latter equality results from Proposition 13 in view of the following computations:

$$
\begin{aligned}
\delta(G, \mu) & =\mu(V(G))-2 \alpha(G, \mu) \\
& =\mu(V(G))-2(\alpha(G, \operatorname{deg}-\mu)+\mu(V(G))-\|G\|) \\
& =2\|G\|-\mu(V(G))-2 \alpha(G, \operatorname{deg}-\mu) \\
& =\operatorname{deg}(V(G))-\mu(V(G))-2 \alpha(G, \operatorname{deg}-\mu) \\
& =\delta(G, \operatorname{deg}-\mu) .
\end{aligned}
$$

For a sequence $\mathcal{T}=\left(T_{1}, T_{2}, \ldots, T_{k}\right)$ of $k$ sets of vertices in a weighted graph $(G, \mu)$, we introduce $3(k-2)$ sets, for $3 \leq j \leq k$ :

$$
B_{j}^{\mathcal{T}}=\left(\cup_{h=1}^{j-1} T_{h}\right) \cap T_{j}, \quad C_{j}^{\mathcal{T}}=\left(\cap_{h=1}^{j-1} T_{h}\right) \backslash T_{j},
$$

and

$$
X_{j}^{\mathcal{T}}=B_{j}^{\mathcal{T}} \cup C_{j}^{\mathcal{T}} .
$$

We will simply write $B_{j}, C_{j}$ and $X_{j}$ when the corresponding sequence $\mathcal{T}$ is clear from the context. The sets $B_{j}$ and $C_{j}$ are disjoint. Moreover, $C_{i} \cap C_{j}=\varnothing$ for $3 \leq i \neq j \leq k$. Here are some lemmas which will be instrumental in showing results concerning the defect of a FDG. 
Lemma 15 Let $(G, \mu)$ be a weighted graph and $\mathcal{T}=\left(T_{1}, T_{2}, \ldots, T_{k}\right)$ be a sequence of subsets of $V(G)$ with $k \geq 2$. Then

$$
\mu\left(\cup_{i=1}^{k} T_{i}\right)+\mu\left(\cap_{i=1}^{k} T_{i}\right)=\sum_{i=1}^{k} \mu\left(T_{i}\right)-\sum_{j=3}^{k} \mu\left(X_{j}\right) .
$$

PROOF. For $k \geq 1$, we let

$$
S_{k}=\mu\left(\cup_{i=1}^{k} T_{i}\right)+\mu\left(\cap_{i=1}^{k} T_{i}\right) .
$$

Then

$$
S_{1}=\mu\left(T_{1}\right)+\mu\left(T_{1}\right), \quad S_{2}=\mu\left(T_{1}\right)+\mu\left(T_{2}\right),
$$

and for $j \geq 3$ :

$$
\begin{aligned}
S_{j}-S_{j-1} & =\mu\left(\cup_{h=1}^{j} T_{h}\right)-\mu\left(\cup_{h=1}^{j-1} T_{h}\right)+\mu\left(\cap_{h=1}^{j} T_{h}\right)-\mu\left(\cap_{h=1}^{j-1} T_{h}\right) \\
& =\mu\left(T_{j}\right)+\mu\left(\left(\cup_{h=1}^{j-1} T_{h}\right) \backslash T_{j}\right)-\mu\left(\cup_{h=1}^{j-1} T_{h}\right)-\left(\mu\left(\cap_{h=1}^{j-1} T_{h}\right)-\mu\left(\cap_{h=1}^{j} T_{h}\right)\right) \\
& =\mu\left(T_{j}\right)-\mu\left(\left(\cup_{h=1}^{j-1} T_{h}\right) \cap T_{j}\right)-\mu\left(\left(\cap_{h=1}^{j-1} T_{h}\right) \backslash T_{j}\right) \\
& =\mu\left(T_{j}\right)-\mu\left(B_{j} \cup C_{j}\right) \\
& =\mu\left(T_{j}\right)-\mu\left(X_{j}\right) .
\end{aligned}
$$

Equation (19) follows from Equations (20)-(21).

For a sequence $\mathcal{T}=\left(T_{1}, T_{2}, \ldots, T_{k}\right)$ of tight sets of a weighted graph $(G, \mu)$, we will need to count separately the edges in the $T_{i}$ 's and in the $X_{j}$ 's. To this aim, we define the 'disjoint unions' of the respective collections of edges:

$$
\mathfrak{T}_{\mathcal{T}}=\cup_{i=1}^{k}\left\{(e, i) \mid e \in E\left(T_{i}\right)\right\}=\cup_{i=1}^{k}\left(E\left(T_{i}\right) \times\{i\}\right),
$$

and

$$
\mathfrak{X}_{\mathcal{T}}=\cup_{j=3}^{k}\left\{(e, j) \mid e \in E\left(X_{j}\right)\right\}=\cup_{j=3}^{k}\left(E\left(X_{j}\right) \times\{j\}\right) .
$$

As for $X_{j}^{\mathcal{T}}=X_{j}$, we simply write $\mathfrak{X}$ and $\mathfrak{T}$ when the corresponding sequence $\mathcal{T}$ is well understood. The total number of tight sets of the weighted graph $(G, \mu)$ under consideration will be denoted as $s$. A scenario of $(G, \mu)$ is a list $\left(T_{1}, T_{2}, \ldots, T_{s}\right)$ of all tight sets of $(G, \mu)$.

Lemma 16 Let $(G, \mu)$ be a FDG and $\mathcal{T}=\left(T_{1}, T_{2}, \ldots, T_{s}\right)$ be a scenario of $(G, \mu)$. Then

$$
\delta(G, \mu)=|\mathfrak{T}|-|\mathfrak{X}|+\sum_{j=3}^{s}\left(w\left(T_{j}\right)-w\left(X_{j}\right)\right) .
$$

Remember that $\|S\|$ denotes the number of edges contained in the set $S$ of vertices. 
PROOF. By Conditions (C3) and (C4) of Proposition 11, we have $\cup_{i=1}^{s} T_{i}=$ $V(G)$ and $\cap_{i=1}^{s} T_{i}=\varnothing$. Lemma 15 then gives

$$
\begin{aligned}
\mu(V(G))= & \mu\left(\cup_{i=1}^{s} T_{i}\right)+\mu\left(\cap_{i=1}^{s} T_{i}\right) \\
= & \sum_{i=1}^{s} \mu\left(T_{i}\right)-\sum_{j=3}^{s} \mu\left(X_{j}\right) \\
= & w\left(T_{1}\right)+\left\|T_{1}||+w\left(T_{2}\right)+\right\| T_{2} \|+ \\
& \sum_{j=3}^{s}\left(w\left(T_{j}\right)+\| T_{j}||\right)-\sum_{j=3}^{s}\left(w\left(X_{j}\right)+\left\|X_{j}\right\|\right) \\
= & 2 \alpha(G, \mu)+\sum_{i=1}^{s}\left\|T_{i}||-\sum_{j=3}^{s}\right\| X_{j} \|+\sum_{j=3}^{s}\left(w\left(T_{j}\right)-w\left(X_{j}\right)\right) \\
= & 2 \alpha(G, \mu)+|\mathfrak{T}|-|\mathfrak{X}|+\sum_{j=3}^{s}\left(w\left(T_{j}\right)-w\left(X_{j}\right)\right) .
\end{aligned}
$$

Building upon the previous lemma, we now show the positivity of the defect of a FDG.

Proposition 17 The defect of any $F D G(G, \mu)$ satisfies $\delta(G, \mu) \geq 1$.

PROOF. Taking again any scenario $\mathcal{T}=\left(T_{1}, T_{2}, \ldots, T_{s}\right)$ of $(G, \mu)$, we refer to Equation (24) in Lemma 16. Because $T_{j}$ is assumed to be a tight set, we have $w\left(T_{j}\right)-w\left(X_{j}\right) \geq 0$. To prove $\delta(G, \mu) \geq 1$, it suffices to show $|\mathfrak{X}| \leq|\mathfrak{T}|+1$. We first exhibit an injective mapping $\varphi$ from $\mathfrak{X}$ to $\mathfrak{T}$, built for any given scenario $\mathcal{T}$. Then for an appropriate choice of the scenario, we show the existence of an element in $\mathfrak{T} \backslash \varphi(\mathfrak{X})$.

Let $(e, j) \in \mathfrak{X}$ where $e=\{u, v\}$. Thus $e \in E\left(X_{j}\right)$ for some $j$ in $\{3,4, \ldots, s\}$, where $X_{j}=B_{j} \cup C_{j}$. This leads to three cases.

Case 1. Assume $e \in E\left(B_{j}\right)$. Because $B_{j} \subseteq T_{j}$, we have $(e, j) \in E\left(T_{j}\right) \times\{j\}$. We then set $\varphi(e, j)=(e, j)$. Here is an illustration: the symbol $*$ indicates where we select $\varphi(e, j)$ (blank entries can be filled randomly with $\in$ or $\notin$ ).

$\begin{array}{cccccccccc} & T_{1} & T_{2} & T_{3} & T_{4} & T_{5} & T_{6} & T_{7} & \ldots & T_{j} \\ u & & & & & \in & & & & \epsilon \\ v & & & \in & & & & & & \in \\ & & & & & & & & & *\end{array}$

Clearly, distinct pairs $(e, j)$ satisfying $e \in B_{j}$ have distinct images. 
Case 2. Assume $e \in E\left(C_{j}\right)$. Because of the definition of $C_{j}$ together with $j \geq 3$, we get $e \in E\left(T_{1}\right)$. We then set $\varphi(e, j)=(e, 1)$.

$\begin{array}{rrrrrrr} & T_{1} & T_{2} & T_{3} & \ldots & T_{j-1} & T_{j} \\ u & \in & \in & \in & \ldots & \epsilon & \notin \\ v & \in & \in & \in & \ldots & \in & \notin \\ & * & & & & & \end{array}$

For each vertex $x$ of $e$, the index $j$ is the least value such that $j \geq 3$ and $x \notin T_{j}$. Consequently, pairs $(e, j)$ with $e \in E\left(C_{j}\right)$ have distinct images by $\varphi$, and all those images differ from the Case 1 images.

Case 3. Assume now $u \in B_{j}$ and $v \in C_{j}$. Exchanging $u$ and $v$ if necessary, this is the last possible case. Again, $j$ is well defined from $e$. We consider subcases according to the value $c$ of the least index $l$ such that $u \notin T_{l}$ (necessarily $c \neq j$ because $u \in B_{j} \subseteq T_{j}$ ).

Subcase 3.1. When $c=1$, we take $r=\min \left\{h \mid e \in E\left(T_{h}\right)\right\}$, and set $\varphi(e, j)=(e, r)$.

$\begin{array}{rrrrrrrrr} & T_{1} & T_{2} & \ldots & T_{r-1} & T_{r} & \ldots & T_{j-1} & T_{j} \\ u & \notin & \notin & \ldots & \notin & \epsilon & & & \in \\ v & \in & \in & \ldots & \in & \in & \ldots & \in & \notin \\ & & & & & * & & & \end{array}$

Because $1<r$ and $e \notin E\left(B_{r}\right)$, we conclude that all of these images are distinct and moreover differ from the images obtained in Cases 1 and 2.

Subcase 3.2. When $2 \leq c<j$, we set $\varphi(e, j)=(e, 1)$.

$\begin{array}{rrrrrrrrr} & T_{1} & T_{2} & \ldots & T_{c-1} & T_{c} & \ldots & T_{j-1} & T_{j} \\ u & \in & \in & \ldots & \in & \notin & & & \in \\ v & \in & \epsilon & \ldots & \in & \epsilon & \ldots & \in & \notin\end{array}$

Distinct pairs falling in this case have distinct images by $\varphi$. For a fixed edge $e$, there cannot exist two distinct pairs $(e, j)$ in $\mathfrak{X}$ such that one fall in Case 2 and the other one in Case 3, so images from the actual subcase differ from those obtained in all preceding cases. 
Subcase 3.3. The only remaining case is when $j<c$. We then set $\varphi(e, j)=$ $(e, 2)$.

$\begin{array}{rrrrrrrrr} & T_{1} & T_{2} & \ldots & T_{j-1} & T_{j} & \ldots & T_{c-1} & T_{c} \\ u & \in & \in & \ldots & \in & \in & \ldots & \in & \notin \\ v & \in & \in & \ldots & \in & \notin & & & \in \\ & & * & & & & & & \end{array}$

Again, distinct pairs in this case have distinct images, and as it is the only case where the pair $(e, 2)$ can be selected, the injectivity of the resulting mapping $\varphi: \mathfrak{X} \rightarrow \mathfrak{T}$ holds for any given scenario $\mathcal{T}$.

Now, let $e \in E(G)$ and choose tight sets $T_{1}, T_{2}$ such that $e \subseteq T_{1}$ and $e \cap T_{2}=\varnothing$ (these tight sets must exist by Proposition $11(\mathrm{C} 3)$ and (C4)). Take any scenario starting with $T_{1}$ and $T_{2}$. Noticing $(e, 1) \in \mathfrak{T} \backslash \varphi(\mathfrak{X})$, we infer $\delta(G, \mu) \geq 1$.

By Proposition 11, Condition (C2), we have $\operatorname{deg}(v)-\mu(v) \geq 1$. Thus the following result strengthens Proposition 17 (but its proof relies on arguments given in the proof of Proposition 17).

Proposition 18 Let $(G, \mu)$ be a FDG and $v$ be any of its vertices. Then $\delta(G, \mu) \geq \operatorname{deg}(v)-\mu(v)$.

In the particular case of stability-critical graphs, Proposition 18 gives $\operatorname{deg}(v) \leq$ $\delta(G)+1$, a theorem of Hajnal (1965) (recalled in Section 6).

PROOF. Let $\mathcal{T}$ be a scenario of $(G, \mu)$ in which the tight sets containing $v$ are listed before those not containing $v$. Let $T_{l}$ be the first tight set of $\mathcal{T}$ which does not contain $v$. The set $N(v)$ of neighbors of $v$ is partitioned into the three following subsets:

$$
\begin{aligned}
& X=\left(N(v) \cap T_{1}\right) \backslash T_{l}, \\
& Y=N(v) \backslash\left(T_{1} \cup T_{l}\right), \\
& Z=N(v) \cap T_{l} .
\end{aligned}
$$

Consider the injective mapping $\varphi: \mathfrak{X} \rightarrow \mathfrak{T}$ built in the proof of Proposition 17 for the scenario $\mathcal{T}$. Then for all $x \in X$ we get either $(\{v, x\}, 1) \in \mathfrak{T} \backslash \varphi(\mathfrak{X})$, in case $x \notin T_{2}$, or $(\{v, x\}, 2) \in \mathfrak{T} \backslash \varphi(\mathfrak{X})$, in case $x \in T_{2}$. Also, for all $y \in Y$, we have $(\{v, y\}, r) \in \mathfrak{T} \backslash \varphi(\mathfrak{X})$, where $r=\min \left\{h \mid\{v y\} \in E\left(T_{h}\right)\right\}$. Consequently, $|\mathfrak{T}|-|\mathfrak{X}| \geq|X \cup Y|=\operatorname{deg}(v)-|Z|$.

Moreover, we observe $v \notin T_{l}, v \in X_{l}$ and $B_{l} \cap N(v)=Z, C_{l} \cap N(v)=\varnothing$. It follows, because $T_{l}$ is tight, $w\left(T_{l}\right)-w\left(X_{l}\right) \geq w\left(X_{l} \backslash\{v\}\right)-w\left(X_{l}\right)=|Z|-\mu(v)$. 
Combining these two observations with Lemma 16 yields (remember that $s$ is the total number of tight sets)

$$
\begin{aligned}
\delta(G, \mu) & =|\mathfrak{T}|-|\mathfrak{X}|+\sum_{j=3}^{s}\left(w\left(T_{j}\right)-w\left(X_{j}\right)\right) \\
& \geq|\mathfrak{T}|-|\mathfrak{X}|+w\left(T_{l}\right)-w\left(X_{l}\right) \\
& \geq \operatorname{deg}(v)-|Z|+|Z|-\mu(v) \\
& =\operatorname{deg}(v)-\mu(v) .
\end{aligned}
$$

Corollary 19 Let $(G, \mu)$ be a FDG and $v$ be any of its vertices. Then $\mu(v) \leq$ $\delta(G, \mu)$.

PROOF. By Proposition 13, $(G, \operatorname{deg}-\mu)$ is also a FDG and by Proposition $14, \delta(G, \mu)=\delta(G, \operatorname{deg}-\mu)$. Applying Proposition 18 to $(G, \operatorname{deg}-\mu)$ and $v$ gives the claim.

Combining Proposition 18 and Corollary 19 gives an upper bound of $2 \delta(G, \mu)$ for the degree of a vertex in a FDG $(G, \mu)$. We conjecture a stronger bound.

Conjecture 20 Let $(G, \mu)$ be a FDG of defect $\delta(G, \mu)$ at least two, and $v$ be any of its vertices. Then $\operatorname{deg}(v) \leq 2 \delta(G, \mu)-1$.

We note that, for each defect $\delta(G, \mu)$ at least 2 , there are examples of FDGs reaching the bound in Conjecture 20. When $(G, \mu)$ is any SFDG (in the sense of Section 7 ), we are able to prove an even stronger result.

Proposition 21 Let $(G, \mu)$ be a SFDG and $v$ be any of its vertices. Then $\operatorname{deg}(v) \leq \delta(G, \mu)+1$.

PROOF. Let $k=\mu(v)$ and $l=\operatorname{deg}(v)-\mu(v)$. By Proposition 11(C2), we have $l \geq 1$. When $k=1$ the claim follows from Proposition 18, so we assume $k \geq 2$. Let $W=\left\{w_{1}, w_{2}, \ldots, w_{k+l}\right\}$ be the set of neighbors of $v$. Let also $\mathcal{T}=\left(T_{1}, \ldots, T_{s}\right)$ be a scenario of $(G, \mu)$ such that the first $l+k+1$ tight sets are specified as follows. For $1 \leq i \leq l+1$, the tight set $T_{i}$ contains $v, w_{1}, w_{2}$, $\ldots, w_{k-1}, w_{k+i-1}$. For $l+2 \leq j \leq l+k$, the tight set $T_{j}$ contains $v, w_{1}, w_{2}, \ldots$, $w_{j-l-2}, w_{j-l}, w_{j-l+1}, \ldots, w_{k+1}$. Finally, we let $T_{l+k+1}$ be a tight set such that $\left\{w_{1}, w_{2}, \ldots, w_{k}\right\} \subseteq T_{l+k+1}$ and $v \notin T_{l+k+1}$. All these tight sets exist by the assumption that $(G, \mu)$ is a SFDG, and they all contain exactly $k$ neighbors of the vertex $v$.

Let now $\varphi: \mathfrak{X} \rightarrow \mathfrak{T}$ be the injective mapping defined for the scenario $\mathcal{T}$ as in the proof of Proposition 17. Then $\left(\left\{v, w_{k+i}\right\}, i+1\right) \in \mathfrak{T} \backslash \varphi(\mathfrak{X})$ can be checked 
for $1 \leq i \leq l$. Also, for $1 \leq j \leq k$, we have $w_{j} \in C_{l+j+1}$ and $v, w_{1}, w_{2}, \ldots$, $w_{j-1}, w_{j+1}, \ldots, w_{k+1} \in B_{l+j+1}$, giving $w\left(X_{l+j+1} \backslash\{v\}\right)-w\left(X_{l+j+1}\right) \geq 1$. Using Lemma 16 we then deduce

$$
\begin{aligned}
\delta(G, \mu) & =|\mathfrak{T}|-|\mathfrak{X}|+\sum_{j=3}^{s}\left(w\left(T_{j}\right)-w\left(X_{j}\right)\right) \\
& \geq|\mathfrak{T}|-|\mathfrak{X}|+\sum_{j=l+2}^{l+k}\left(w\left(T_{j}\right)-w\left(X_{j}\right)\right) \\
& \geq|\mathfrak{T}|-|\mathfrak{X}|+\sum_{j=l+2}^{l+k}\left(w\left(X_{j} \backslash\{v\}\right)-w\left(X_{j}\right)\right) \\
& \geq|\mathfrak{T}|-|\mathfrak{X}|+k-1 \\
& \geq l+k-1=\operatorname{deg}(v)-1 .
\end{aligned}
$$

The defect was shown to be a useful invariant for the investigation of stabilitycritical graphs, in particular for attempting to classify these graphs (see Section 6). In view of the current section, the same assertion applies also to the weighted case. We now make a first elementary step in the classification of FDGs.

Proposition 22 FDGs of defect one are the odd cycles with the weight function 11 .

PROOF. Let $(G, \mu)$ be a FDG of defect one. By Proposition $11(\mathrm{C} 2)$ and Corollary 19, it follows that $\mu=11$. By Proposition 3, the graph $G$ must be a stability-critical graph of defect one, that is, an odd cycle.

We are currently investigating FDGs of defect two. Similarly as for stabilitycritical graphs (see Section 6), one could hope that all FDGs of a fixed defect are generated by repeatedly applying some well-defined construction to a finite number of basic graphs. We were able to design such a generation procedure only for SFDGs of defect two, and are presently trying to extend the findings to all FDGs of defect two. The case of a larger defect remains to be investigated.

\section{Conclusion}

The whole family of fence inequalities for the linear ordering polytope have been subsumed to a general form of facet-defining inequalities. Called the graphical inequalities, the latters are built from specific weighted graphs. The weighted graphs which define facets in this way generalize stability-critical 
graphs. They are investigated, in particular with regard to their defect. We point out that Corollary 9 and Proposition 13 imply that connected stabilitycritical graphs produce a facet not only as in Koppen (1995) (that is, taken with all weights equal to 1 ), but also when the weight of any vertex $v$ is set to the degree of $v$ minus 1 .

We mention that there are facet-defining inequalities for the linear ordering polytope which are not graphical, for instance the Möbius inequalities: see, e.g., Grötschel et al. (1985a), Borndörfer and Weismantel (2000) and Fiorini $(2005)$

\section{References}

Andrásfai, B., 1967. On critical graphs. In: Theory of Graphs (Internat. Sympos., Rome, 1966). Gordon and Breach, New York, pp. 9-19.

Beineke, L. W., Harary, F., Plummer, M., 1967. On the critical lines of a graph. Pacific J. Math. 22, 205-212.

Block, H. D., Marschak, J., 1960. Random orderings and stochastic theories of responses. In: Contributions to probability and statistics. Stanford Univ. Press, Stanford, Calif., pp. 97-132.

Bolotashvili, G., 1987. A class of facets of the permutation polytope and a method for constructing facets of the permutation polytope. Tech. Rep. 3403-B87 and N3405-B87, VINITI (in Russian).

Bolotashvili, G., Kovalev, M., Girlich, E., 1999. New facets of the linear ordering polytope. SIAM J. Discrete Math. 12, 326-336.

Borndörfer, R., Weismantel, R., 2000. Set packing relaxations of some integer programs. Math. Program. 88 (Ser. A), 425-450.

Christophe, J., Doignon, J.-P., Fiorini, S., 2003. Counting biorders. Journal of Integer Sequences 6, article 03.4.3, 10 pages.

Christophe, J., Doignon, J.-P., Fiorini, S., 2004. The biorder polytope. Order $21,61-82$.

Cohen, M., Falmagne, J.-C., 1978. Random scale representation of binary choice probabilities: A counterexample to a conjecture of Marschak. Tech. rep., Department of Psychology, New York University, New York, unpublished manuscript.

Cohen, M., Falmagne, J.-C., 1990. Random utility representation of binary choice probabilities: a new class of necessary conditions. J. Math. Psych. 34, 88-94.

Diestel, R., 2000. Graph theory, 2nd Edition. Springer-Verlag, New York.

Doignon, J.-P., Ducamp, A., Falmagne, J.-C., 1984. On realizable biorders and the biorder dimension of a relation. Journal of Mathematical Psychology 28, 73-109. 
Ducamp, A., Falmagne, J. C., 1969. Composite measurement. Journal of Mathematical Psychology 6, 359-390.

Erdős, P., Gallai, T., 1961. On the minimal number of vertices representing the edges of a graph. Magyar Tud. Akad. Mat. Kutató Int. Közl. 6, 181-203.

Erdős, P., Hajnal, A., Moon, J. W., 1964. A problem in graph theory. Amer. Math. Monthly 71, 1107-1110.

Falmagne, J.-C., 1978. A representation theorem for finite random scale systems. J. Math. Psych. 18, 52-72.

Falmagne, J.-C., 1979. Errata: "A representation theorem for finite random scale systems" (J. Math. Psych. 18 (1978), no.1, 52-72). J. Math. Psych. $19,219$.

Fiorini, S., 2001. Determining the automorphism group of the linear ordering polytope. Discrete Appl. Math. 112, 121-128.

Fiorini, S., 2004. A short proof of a theorem of Falmagne. J. Math. Psych. 48, $80-82$.

Fiorini, S., 2005. How to recycle your facets. Tech. rep., submitted.

Fishburn, P. C., 1990. Binary probabilities induced by rankings. SIAM J. Discrete Math. 3, 478-488.

Gilboa, I., 1990. A necessary but insufficient condition for the stochastic binary choice problem. J. Math. Psych. 34, 371-392.

Grötschel, M., Jünger, M., Reinelt, G., 1985a. Facets of the linear ordering polytope. Math. Programming 33, 43-60.

Grötschel, M., Jünger, M., Reinelt, G., 1985b. On the acyclic subgraph polytope. Math. Programming 33, 28-42.

Guilbaud, G., 1953. Sur une difficulté de la théorie du risque. In: Econométrie. Vol. 1952 of Colloques Internationaux du Centre National de la Recherche Scientifique, no. 40, Paris. Centre de la Recherche Scientifique, Paris, pp. 19-25; discussion, pp. 25-28.

Guttman, L., 1944. A basis for scaling quantitative data. American Sociological Review 9, 139-150.

Hajnal, A., 1965. A theorem on $k$-saturated graphs. Canad. J. Math. 17, 720724.

Harary, F., Plummer, M. D., 1967. On indecomposable graphs. Canad. J. Math. 19, 800-809.

Karp, R. M., 1972. Reducibility among combinatorial problems. In: Complexity of computer computations (Proc. Sympos., IBM Thomas J. Watson Res. Center, Yorktown Heights, N.Y., 1972). Plenum, New York, pp. 85-103.

Koppen, M., 1991. Random utility representation of binary choice probabilities. In: Doignon, J.-P., Falmagne, J.-C. (Eds.), Mathematical psychology: current developments. Springer Verlag, New York, pp. 181-201.

Koppen, M., 1995. Random utility representation of binary choice probabilities: critical graphs yielding critical necessary conditions. J. Math. Psych. $39,21-39$.

Leung, J., Lee, J., 1994. More facets from fences for linear ordering and acyclic subgraph polytopes. Discrete Appl. Math. 50, 185-200. 
Lipták, L., Lovász, L., 2000. Facets with fixed defect of the stable set polytope. Math. Program. 88, 33-44.

Lipták, L., Lovász, L., 2001. Critical facets of the stable set polytope. Combinatorica 21, 61-88.

Lovász, L., 1978. Some finite basis theorems on graph theory. In: Combinatorics (Proc. Fifth Hungarian Colloq., Keszthely,1976), Vol. II. Vol. 18 of Colloq. Math. Soc. János Bolyai. North-Holland, Amsterdam, pp. 717-729.

Lovász, L., 1993. Combinatorial Problems and Exercises, 2nd Edition. NorthHolland Publishing Co., Amsterdam.

Lovász, L., Plummer, M. D., 1986. Matching Theory. Vol. 121 of NorthHolland Mathematics Studies. North-Holland Publishing Co., Amsterdam, Annals of Discrete Mathematics, 29.

McLennan, A., 1990. Binary stochastic choice. In: Chipman, J., McFadden, D., Richter, M. (Eds.), Preferences, Uncertainty, and Optimality. Westview Press, Boulder, CO, pp. 187-202.

Megiddo, N., 1977. Mixtures of order matrices and generalized order matrices. Discrete Math. 19, 177-181.

Plummer, M. D., 1967. On a family of line-critical graphs. Monatsh. Math. $71,40-48$.

Riguet, J., 1951. Les relations de Ferrers. Comptes Rendus des Séances de l'Académie des Sciences (Paris) 232, 1729-1730.

Sewell, E. C., Trotter, Jr., L. E., 1993. Stability critical graphs and even subdivisions of $K_{4}$. J. Combin. Theory Ser. B 59, 74-84.

Sewell, E. C., Trotter, Jr., L. E., 1995. Stability critical graphs and ranks facets of the stable set polytope. Discrete Math. 147, 247-255.

Suck, R., 1992. Geometric and combinatorial properties of the polytope of binary choice probabilities. Math. Social Sci. 23, 81-102.

Surányi, L., 1975a. Large $\alpha$-critical graphs with small deficiency. On linecritical graphs. II. Studia Sci. Math. Hungar. 10, 397-412 (1978).

Surányi, L., 1975b. On line critical graphs. In: Infinite and finite sets (Colloq., Keszthely, 1973; dedicated to P. Erdős on his 60th birthday), Vol. III. NorthHolland, Amsterdam, pp. 1411-1444. Colloq. Math. Soc. Jańos Bolyai, Vol. 10.

Surányi, L., 1978. A note on a conjecture of Gallai concerning $\alpha$-critical graphs. In: Combinatorics (Proc. Fifth Hungarian Colloq., Keszthely, 1976), Vol. II. Vol. 18 of Colloq. Math. Soc. János Bolyai. North-Holland, Amsterdam, pp. $1065-1074$.

Surányi, L., 1980. On a generalization of line-critical graphs. Discrete Math. 30, 277-287.

Wessel, W., 1968. Eine Methode zur Konstruktion von kanten-p-kritischen Graphen. In: Beiträge zur Graphentheorie (Kolloquium, Manebach, 1967). Teubner, Leipzig, pp. 207-210.

Wessel, W., 1970a. Kanten-kritische Graphen mit der Zusammenhangszahl 2. Manuscripta Math. 2, 309-334.

Wessel, W., 1970b. On the problem of determining whether a given graph is 
edgecritical or not. In: Combinatorial theory and its applications, III (Proc. Colloq., Balatonfüred, 1969). North-Holland, Amsterdam, pp. 1123-1139.

Wessel, W., 1975. A first family of edge-critical wheels. Period. Math. Hungar. 6, 229-233.

Wessel, W., 1978. A second family of edge-critical wheels. In: Combinatorics (Proc. Fifth Hungarian Colloq., Keszthely, 1976), Vol. II. Vol. 18 of Colloq. Math. Soc. János Bolyai. North-Holland, Amsterdam, pp. 1123-1145.

Zhu, Q. C., 1989. The structure of $\alpha$-critical graphs with $|V(G)|-2 \alpha(G)=3$. In: Graph theory and its applications: East and West (Jinan, 1986). Ann. New York Acad. Sci. 576, 716-722. 